ARTICLE

DOI: $10.1038 / \mathrm{s} 41467-017-00545-0$

OPEN

\title{
Bio-degradable highly fluorescent conjugated polymer nanoparticles for bio-medical imaging applications
}

\author{
Tatjana Repenko', Anne Rix², Simon Ludwanowski , Dennis Go ${ }^{1}$, Fabian Kiessling ${ }^{2}$, \\ Wiltrud Lederle ${ }^{2} \&$ Alexander J.C. Kuehne (iD ${ }^{1}$
}

Conjugated polymer nanoparticles exhibit strong fluorescence and have been applied for biological fluorescence imaging in cell culture and in small animals. However, conjugated polymer particles are hydrophobic and often chemically inert materials with diameters ranging from below $50 \mathrm{~nm}$ to several microns. As such, conjugated polymer nanoparticles cannot be excreted through the renal system. This drawback has prevented their application for clinical bio-medical imaging. Here, we present fully conjugated polymer nanoparticles based on imidazole units. These nanoparticles can be bio-degraded by activated macrophages. Reactive oxygen species induce scission of the conjugated polymer backbone at the imidazole unit, leading to complete decomposition of the particles into soluble low molecular weight fragments. Furthermore, the nanoparticles can be surface functionalized for directed targeting. The approach opens a wide range of opportunities for conjugated polymer particles in the fields of medical imaging, drug-delivery, and theranostics.

\footnotetext{
${ }^{1}$ DWI-Leibniz Institute for Interactive Materials, RWTH Aachen University, Forckenbeckstraße 50, 52076 Aachen, Germany. ${ }^{2}$ Institute for Experimental Molecular Imaging, University Clinic and Helmholtz Institute for Biomedical Engineering, RWTH Aachen University, Pauwelsstraße 30, 52074 Aachen, Germany. Correspondence and requests for materials should be addressed to W.L. (email: wlederle@ukaachen.de) or to A.J.C.K. (email: kuehne@dwi.rwth-aachen.de)
} 
C onjugated polymer nanoparticles (CPNs) are compelling probes with prospective use in bio-medical imaging ${ }^{1-4}$. The particles can be applied for staining cells ${ }^{5}$ and tissue 6 for fluorescence-based imaging techniques such as microscopy and tomography ${ }^{7}$. CPNs are hydrophobic, therefore, quickly taken up by cells and they exhibit low cytotoxicity ${ }^{8}$. Due to their $\pi$-conjugated electron system, these organic semiconductor particles are strongly fluorescent and the emission color can be tuned across the entire visible spectrum into the near infrared (NIR), simply by adjusting the co-monomer composition ${ }^{9}$. Furthermore, CPNs also show excellent performance as photoacoustic contrast agents and for image-guided photodynamic and photothermal therapy $^{7}, 10,11$. Because the entire CPN consists of semiconducting material, their fluorescence is extremely photostable, allowing detection over long observation times ${ }^{5,12}$. Furthermore, CPNs can be surface functionalized with biological recognition entities such as peptides ${ }^{12}$, glycans ${ }^{13}$, or antibodies ${ }^{14}$. Such surface functionalized nanoparticles are applied to specifically target bio-molecular motifs expressed on the membrane of cells in pathological tissue for example in cancerous tumors ${ }^{15}, 16$. CPNs can be produced by a variety of methods ranging from dispersion polymerization protocols ${ }^{12,17-21}$ to post-polymerization miniemulsification $^{22,23}$ or nanoprecipitation techniques, ${ }^{5,24,25}$. While direct polymerization methods deliver particles in the range of 200 to $2 \mu \mathrm{m}$, post-polymerization techniques can also produce much smaller nanoparticles (often termed dots) with diameters below $50 \mathrm{~nm}$. However, with respect to in vivo and especially clinical applications the critical diameter for nanoparticles allowing for renal clearance is around $5-6 \mathrm{~nm}^{26}$. Particles in the blood stream above this size-limit will be ingested by macrophages eventually leading to accumulation in the spleen or liver ${ }^{27}$. If the particles cannot be degraded by macrophages, they will accumulate and persist in organs and might cause unforeseeable adverse effects ${ }^{28}$. To circumvent this problem, colloidal entities such as liposomes, micelles, or degradable polymers are commonly applied in vivo, which can decompose into their small molecular building blocks ${ }^{29}$. Several polymer particles have been developed with a variety of degradable units, which allow for chain scission ${ }^{30}$. The triggers for chain scission range from acidic environments where esters and hydrazones are hydrolyzed to incorporated peptide sequences, which can be cleaved enzymatically $^{31}$. The intact entity is much larger in dimension than the pore-diameter of the kidney membranes and, therefore, provides in principle long circulation times in the body. The degraded products have considerably lower molecular weights and can easily be cleared from the body through the renal membranes.
However, such colloidal entities usually suffer from a lack in satisfactory fluorescence, sufficient imaging properties, and effectual contrast. None of the classical degradable polymers provide $\pi$-conjugation and the resulting polymer particles are dielectric in nature. On the contrary, CPNs are generally nondegradable due to their inert and carbon-based $\pi$-conjugated architecture. The absence of degradability in conjugated polymer particle systems prevents their safe in vivo application. This deficiency impedes their translation into the clinic and the utilization of a class of highly potent fluorescent and photoacoustically active materials, which could enhance contrast, obviate photo-bleaching and augment signal-to-noise ratios for biological and medical imaging technologies 1,3 . To achieve bio-degradability in fluorescent CPNs, we have to apply a conjugated moiety, which can be cleaved. However, such units are currently not known and unavailable for conjugated polymers. When looking at nature, biological light emitting units often contain an imidazole ring. Examples for these molecules are coelenterazine, vargulin ${ }^{32,33}$, and the green fluorescent protein $\mathrm{GFP}^{34}$. The light emitting unit is generated by auto-oxidation resulting in fusion of aminoacids (such as $\pi$-conjugated phenylalanine, tyrosine with non-conjugated serine, and glycine) producing the imidazole unit. The emitting units are biodegradable through further oxidation and scission of the imidazole ring, affording again aminoacids as well as amides, which can be further metabolized ${ }^{35-37}$. This degradative oxidation can also be induced by reactive oxygen species (ROS), which are generated for example by activated macrophages ${ }^{38}$.

Here, we develop a CPN system based on imidazole units, affording highly fluorescent and biodegradable particles. The resulting polymer chains are fully conjugated along the backbone. The particles decompose upon exposure to ROS such as hydrogen peroxide at concentrations that are relevant for in vivo applications ${ }^{39}$. We show how the particles are degraded by activated macrophages in cell culture. The resulting small molecular degradation products are water soluble. The degradable particles can also be surface functionalized with bio-medical homing devices to allow for targeted delivery of the conjugated polymer imaging probe. The development of bio-degradable CNPs presents a stepping-stone for rational in vivo application of conjugated polymer particles and potential transfer into the clinic.

\section{Results}

Synthesis of degradable CPNs. To incorporate potentially degradable imidazole units into our CPNs, we apply diiodo- $N$ - a

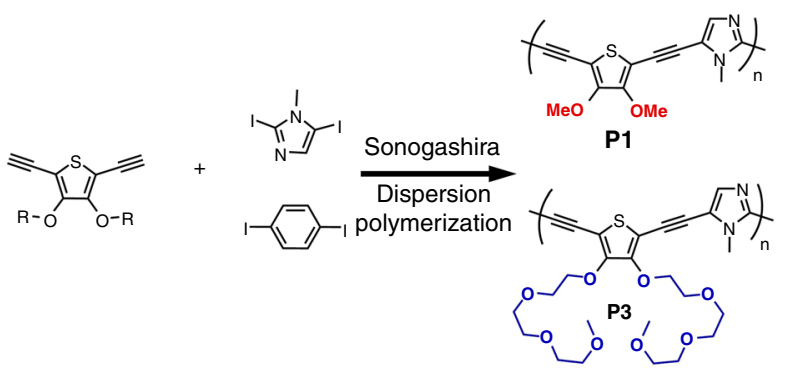

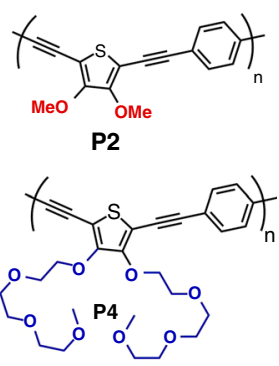

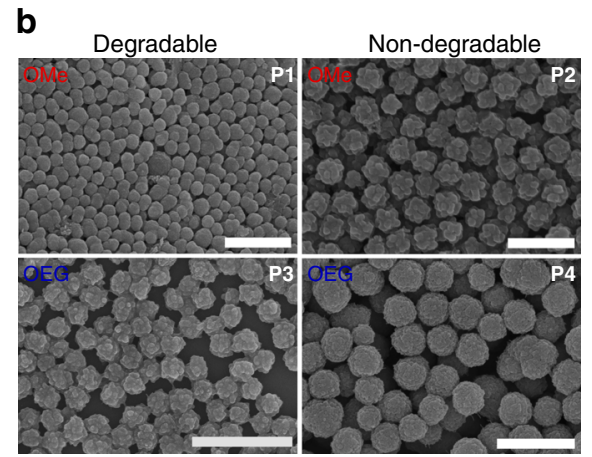

Fig. 1 Conjugated polymer nanoparticle synthesis. Sonogashira dispersion polymerization of thiophene monomers with imidazole monomers to produce degradable particles and polymerization with benzene monomers to obtain non-degradable particles. a The thiophene monomer carries either methoxy- (OMe, red) or oligoethyleneoxy- (OEG, blue) side-groups to tune the particle morphology, provide stability for the particles in water and improve the solubility of the degradation products. b Scanning electron micrograph of degradable (P1 and $\mathbf{P 3}$ ) and non-degradable (P2 and $\mathbf{P 4 )}$ particles. The scale bar represents $1 \mu \mathrm{m}$ 
methyl-imidazole. CNPs are produced via Sonogashira dispersion polymerization with an acetylene functionalized thiophene co-monomer to produce uniform particles of the respective fully $\pi$-conjugated polymers (see Fig. 1). Alternatively, the functional groups on the monomers can be interchanged. Sonogashira-based step-growth dispersion polymerization has been developed by us and reported previously ${ }^{12}$. In short, particle preparation involves polymerization of the monomers in 1-propanol. This solvent is good for the monomers but poor for the resulting polymers. When a critical molecular weight is reached particle nuclei precipitate from solution and coalesce until they are stabilized by added Triton X-45 and poly(vinylpyrrolidone-co-vinylacetate) preventing further particle aggregation. These stabilized nuclei act as seeds for subsequently generated polymer chains reaching the critical molecular weight $(\sim 15 \mathrm{kDa})$, which condensate onto the particles ${ }^{40}$. This way the particles grow until they reach their final size of a few hundred nanometers in diameter ${ }^{18}$. The size of the particles can be controlled by tuning of the applied monomer concentration. The thiophene monomer unit carries either methoxy- (OMe for P1) or oligoethyleneoxy (OEG for P3) sidegroups to direct the particle morphology, provide stability for the particles in water, and improve the solubility of the resulting degradation products. We apply monomer concentrations to aim for particle sizes at the bottom of the accessible size scale. The variation of the alkoxy periphery entails small differences in the final sizes. We observe particle diameters between 210 and 250 $\mathrm{nm}$ (determined via scanning electron microscopy (SEM) image analysis of at least 100 particles). The particles are uniform in size; however, they exhibit rough surfaces as can be seen when imaging the particles in dry state by SEM (see Fig. 1). The rough surface is probably a remnant of the initial nucleation coalescence phase during particle synthesis as well as different tendencies of the rigid backbones and different side groups to crystallize during the condensation phase of the dispersion polymerization ${ }^{41}$. To investigate whether the particles are stable in dispersion, we perform dynamic light scattering (DLS) in water. The CPNs do not aggregate and are individually dispersed. The DLS data corresponds relatively well with the SEM results and gives hydrodynamic diameters of $290 \mathrm{~nm}$ for P1 and $282 \mathrm{~nm}$ for P3 and dispersities of only 5\%, respectively (see Supplementary Table 1 and Supplementary Figs. 13 and 15). We also produce particles by copolymerizing $p$-diiodobenzene instead of the degradable imidazole unit to obtain non-degradable particles P2 and $\mathbf{P 4}$ as controls (see Fig. 1). These particles exhibit hydrodynamic diameters of $492 \mathrm{~nm}$ for P2 and $574 \mathrm{~nm}$ for P4 (Supplementary Table 1 and Supplementary Figs. 14 and 16).

Degradable imidazole CPNs. To test whether they are degradable, we expose $\mathbf{P 1}$ particles to hydrogen peroxide $\left(\mathrm{H}_{2} \mathrm{O}_{2}\right)$ at $20 \mu \mathrm{M}$ in water for $48 \mathrm{~h}$. During this time the turbid dispersion turns transparent. In agreement with the previously described imidazole oxidation ${ }^{35}$, we expect the particles to decompose into methylaminoacidyl- and amide terminated 2,5-diethynylene-3,4methoxythiophene (see Fig. 2a). When we perform ${ }^{1} \mathrm{H}-\mathrm{NMR}$ spectroscopy of the degradation products, we indeed find the respective signals for the methine hydrogen, the amines and the acid hydroxyl group proving scission of the imidazole ring (see Fig. 2a and Supplementary Fig. 9). When we expose the P1 particles to $20 \mu \mathrm{M}$ of $\mathrm{H}_{2} \mathrm{O}_{2}$ for only a few minutes and deposit a

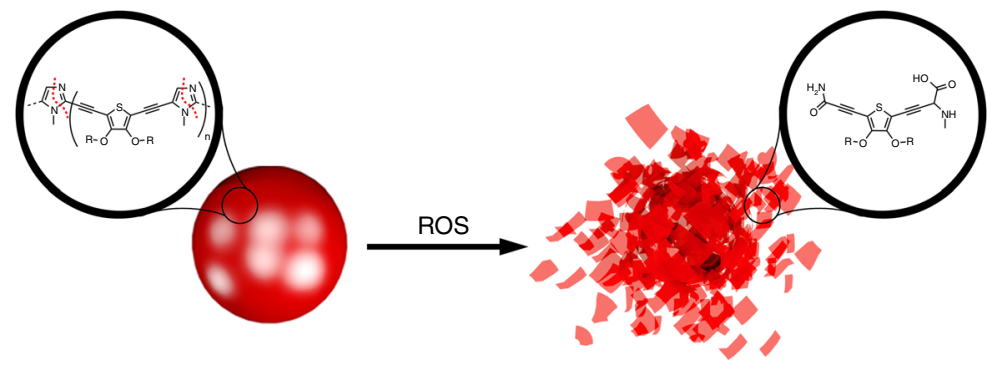

d

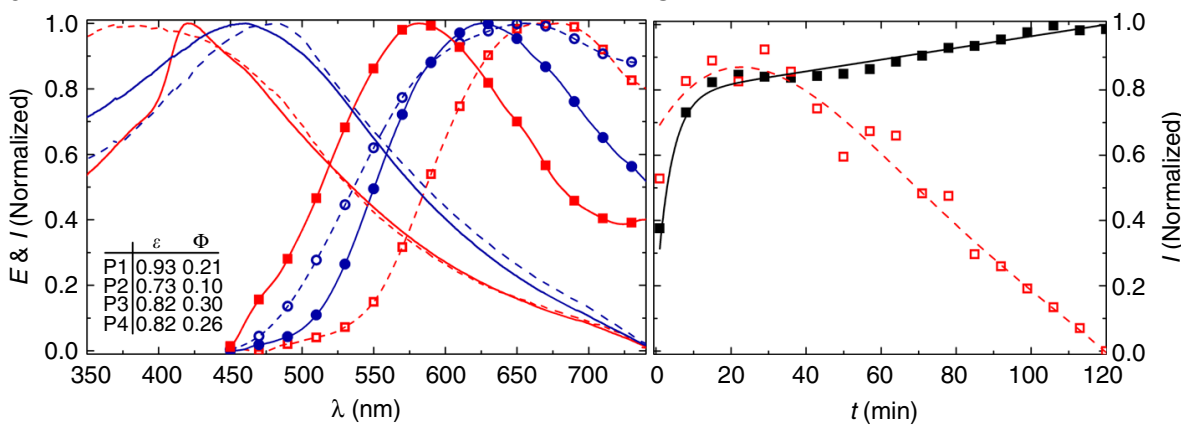

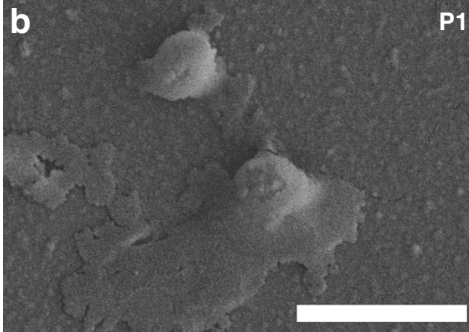

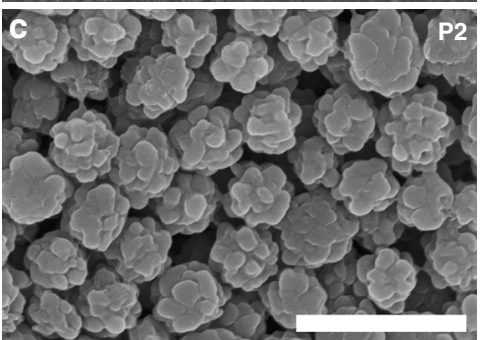

Fig. 2 Characterization of the conjugated polymer nanoparticles. a Degradative imidazole oxidation of particle by reactive oxygen species to decompose into methylaminoacid and amide terminated 2,5-diethynylene-3,-4-methoxythiophene. b Scanning electron micrographs of partially degraded P1 after treatment with $\mathrm{H}_{2} \mathrm{O}_{2}(20 \mu \mathrm{M}$ in water). The scale bar represents $1 \mu \mathrm{m}$. c Scanning electron micrograph of intact non-degradable P2 after treatment with $\mathrm{H}_{2} \mathrm{O}_{2}$ (20 $\mu \mathrm{M}$ in water). The scale bar represents $1 \mu \mathrm{m}$. d Extinction and photoluminescence spectra of P1 (dashed red, PL: open squares), P2 (solid red, PL: squares), P3 (dashed blue, PL open circles), and P4 (solid blue, PL: circles), all excited at 400 nm. The degradable particles (P1, P3) show a broad emission tail reaching into the near $I R$ with maxima at around $670 \mathrm{~nm}$. The non-degradable control particles (P2, P4) are blue shifted with emission maxima at $630 \mathrm{~nm}$ (P2) and $570 \mathrm{~nm}$ (P4). The table inset gives extinction coefficients $\varepsilon$ and photoluminescence quantum yields $\Phi$ for P1-P4. e Fluorescence intensity of the conjugated particles (at a loading of $0.3 \mathrm{mg} / \mathrm{ml}$ ) P1 (red) and $\mathbf{P 2}$ (black) versus exposure time to $\mathrm{H}_{2} \mathrm{O}_{2}(0.3 \mathrm{M}$ ). The intensities increase by about $40 \%$ caused by a photo-brightening effect. After 20 min, a decrease in the fluorescence intensity of $\mathbf{P 1}$ is apparent, caused by the degradation of $\mathbf{P 1}$. This decrease in fluorescence intensity is not observed in $\mathbf{P 2}$ particles 
and dry them on a surface for SEM analysis we can capture particles, which are only partially degraded (see Fig. 2b). When performing the same experiment with the non-degradable P2 particles the shape remains intact ( $c f$. Figs. 1 and 2c).

The CNPs exhibit fluorescence in the red spectrum. The imidazole particles (P1, P3) are deep red with emission maxima at around $670 \mathrm{~nm}$ and a broad emission tail reaching into the NIR. By contrast, the control particles are blue shifted in their emission, with maximum intensities of $630 \mathrm{~nm}$ for P2 and at $570 \mathrm{~nm}$ for P4 (see Fig. 2d). This shift is due to different intramolecular electronic effects of the phenylene versus the more electron rich imidazole unit. The methoxy- and OEG functionalization of the polymer chains leads to different molecular aggregation behavior. The OEG side-chains in P3 will induce $J$-aggregates, with a red-shifted fluorescence maximum compared to the methoxy-functionalized P1 particles ${ }^{42,43}$. We can also follow the degradation of the CPNs by looking at the decay of the fluorescence maximum, while exposing the particles to a solution of $\mathrm{H}_{2} \mathrm{O}_{2}$.

The degradable particles $\mathbf{P 1}$ are exposed to a $1 \mathrm{wt} \%(0.3 \mathrm{M})$ solution of $\mathrm{H}_{2} \mathrm{O}_{2}$ and the fluorescence maximum is recorded over $2 \mathrm{~h}$ (see Fig. 2e). We first observe an increase of fluorescence until $20 \mathrm{~min}$ into the measurement. Thenceforth, the fluorescence of the particles deteriorates linearly (see red data in Fig. 2e). While the linear decay is expected, the apparent intensity increase by about $40 \%$ in the beginning of the measurement is surprising. The increase can either be explained by the fact that through degradation, single chains or lose ends are fully solvated and fluoresce at a higher quantum yield because of reduced intermolecular quenching. Alternatively, the fluorescence intensity increase is caused by a photo-brightening effect, which is induced by the measurement beam and known to occur in conjugated polymers ${ }^{44}$. The latter mechanism seems to be the correct explanation as we also see the photo-brightening effect in the non-degradable P2 control particles (see black data in Fig. 2e). In addition to the fluorescence spectroscopy experiments we also perform confocal fluorescence microscopy to image the CPNs over the course of degradation and rule out unexpected bleaching effects, which could cause the fluorescence decay. The particles exhibit Brownian motion, which is why we image immobilized particles that adhere to the bottom substrate of our imaged specimen (see Fig. 3a and Supplementary Movie 1). Also, here we see the photo-brightening effect, when we plot the fluorescence of the entire field of view versus time (see Supplementary Fig. 12). At the same time we look at the CPNs with bright-field microscopy to also obtain information about aggregates, which are not in the confocal plane (see Fig. $3 b$ and Supplementary Movie 1). It is clearly visible that over the course of only $12 \mathrm{~min}$, the fluorescent particle aggregates disintegrate and the individual particles disappear (see Fig. 3a). Also in the bright-field, the disintegration can be followed until there are no more particles visible. This study proves that the degradation occurs throughout the entire sample and not just at the interface (see Fig. 3b). When we repeat the experiments with non-degradable $\mathbf{P} 2$ particles, the particles remain intact over the entire time of observation. This clearly indicates that the conjugated polymers are cleaved at the imidazole moiety (see Supplementary Movie 2).

Biodegradation of CPNs. Having proven that fully conjugated polymer particles with imidazole units in the backbone can be degraded using $\mathrm{H}_{2} \mathrm{O}_{2}$ at concentrations beyond biologically relevant levels; we now want to look at bio-degradability of our particles. To investigate biodegradability we add our particles to J774A.1 macrophages in cell culture. The macrophages are exposed to lipopolysaccharides (LPS), which is a well-known stimulant to activate the macrophages to produce ROS, such as $\mathrm{H}_{2} \mathrm{O}_{2}$. The macrophages are stained using a CellTracker ${ }^{\mathrm{Tm}}$ Blue CMHC dye for better visualization and incubated with our particles for two hours before transferal to the confocal microscope, where we image and follow individual cells with few ingested particles over a period of 3 days (see Fig. 4 and Supplementary Fig. 10). On the confocal microscope the cells are cultivated in FluoroBrite ${ }^{\mathrm{TM}}$ medium, which is low in autofluorescence, in a $\mathrm{CO}_{2}$ rich atmosphere of $5 \%$ and at a temperature of $37.5^{\circ} \mathrm{C}$. For all samples we observe that the macrophages appear healthy for the first $12 \mathrm{~h}$ with some macrophages starting to have disrupted cell membranes at a time of $24 \mathrm{~h}$. At the applied concentration $(4 \mu \mathrm{g} / \mathrm{ml})$ the degradation products do not seem to be toxic, at least not over the course of the experiment. To validate these hypotheses, we perform a cytotoxicity study under more controlled cell culture conditions (i.e., in a dedicated incubator, with controlled humidity and $\mathrm{CO}_{2}$ level). We compare the FluoroBrite ${ }^{\mathrm{Ts}}$ medium to standard DMEM medium and test also for higher particle concentrations of up to $100 \mu \mathrm{g} / \mathrm{ml}$ (see Supplementary Fig. 11). We determine relative cell
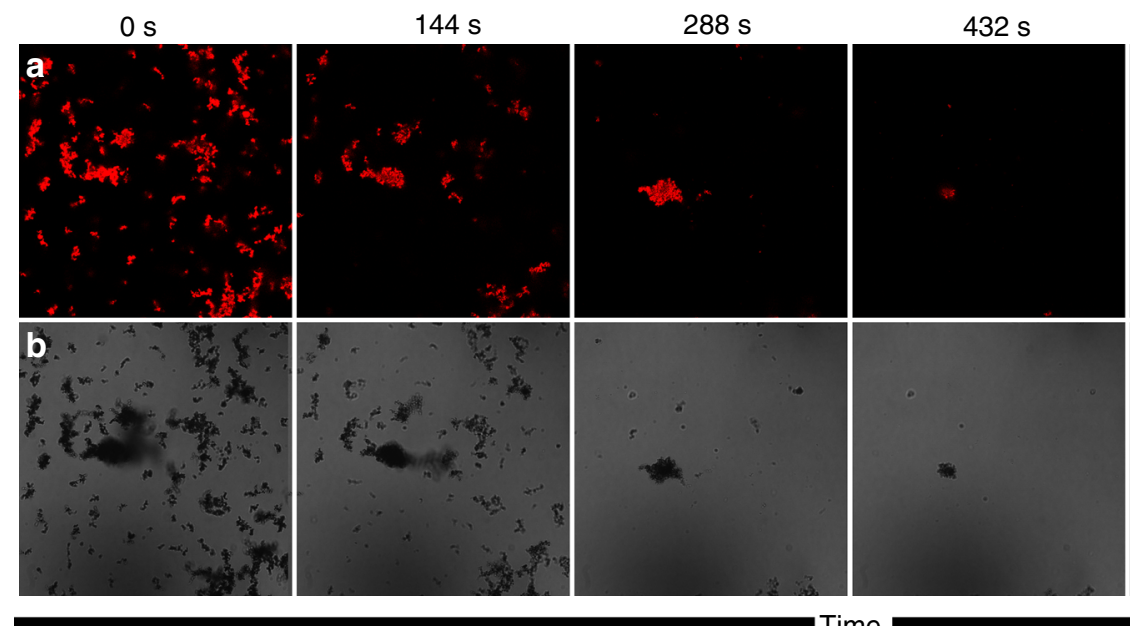

$576 \mathrm{~s}$

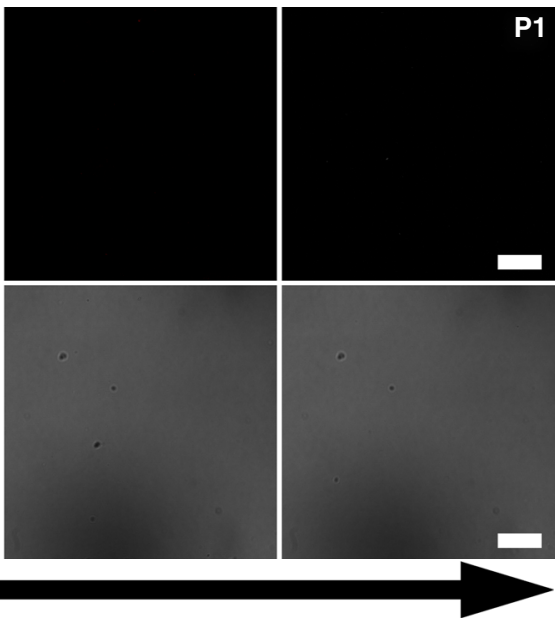

Fig. 3 Degradation of the imidazole carrying conjugated polymer nanoparticles. a Confocal fluorescence microscopy images and $\mathbf{b}$ bright-field images of degradable particles $\mathbf{P 1}$ exposed to an aqueous $3 \mathrm{M} \mathrm{H}_{2} \mathrm{O}_{2}$ solution. After $12 \mathrm{~min}$ the fluorescent particle aggregates disintegrate and individual particles disappear. The scale bars represent $20 \mu \mathrm{m}$ 

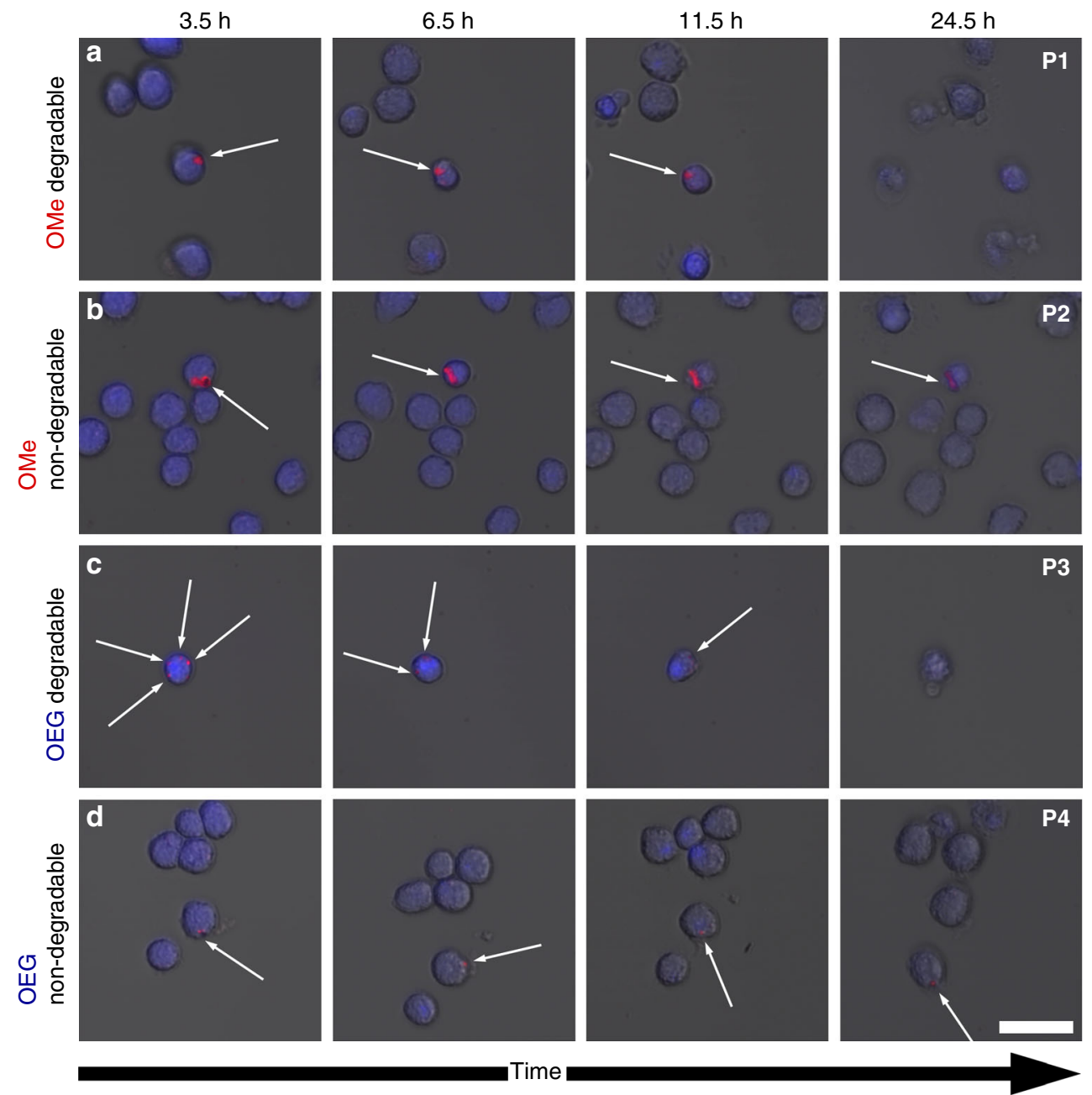

Fig. 4 Confocal fluorescence microscopy of macrophages (stained in blue) incubated with degradable and non-degradable particles. The macrophages are exposed to lipopolysaccharides (LPS) to activate the macrophages to produce ROS (e.g., $\mathrm{H}_{2} \mathrm{O}_{2}$ ). The fluorescent particles are highlighted by white arrows (Extended time series in Supplementary Fig. 10). a The OMe functionalized degradable $\mathbf{P 1}$ particles shrink and the fluorescence signal disappears after 11.5 h. b The OMe functionalized non-degradable $\mathbf{P 2}$ particles. c The OEG functionalized $\mathbf{P 3}$ particles are degraded and the fluorescence signal disappears after $11.5 \mathrm{~h}$. d The OEG functionalized $\mathbf{P 4}$ particles as well as the $\mathbf{P 2}$ particles in $\mathbf{b}$ do not disintegrate and the fluorescence is visible until the end of the experiment. The faster degradation is caused by the improved solubility in aqueous medium. The white arrows indicate where particles are present. The scale bar represents $35 \mu \mathrm{m}$

viabilities with respect to LPS activated macrophages incubated in FluoroBrite ${ }^{\mathrm{me}}$, which have not been exposed to degradable particles (as a control). We observe good relative cell viabilities between 80 and $100 \%$ for macrophages exposed to $5 \mu \mathrm{g} / \mathrm{ml}$ of particles over a period of up to $24 \mathrm{~h}$, independent of their alkoxyfunctionalization or their degradability (see Fig. $5 \mathrm{a}$ and Supplementary Fig. 11b-d). This concentration is similar to the one used during the confocal microscopy study $(4 \mu \mathrm{g} / \mathrm{ml})$. At high particle concentrations of $100 \mu \mathrm{g} / \mathrm{ml}$ the viability of macrophages with ingested degradable particles is lower than for the nondegradable particles. This suggests some toxicity of the degradation products at high concentrations. All macrophages enter apoptosis after about $24 \mathrm{~h}$ into the experiment. This effect is not due to the particles but can be explained by the high concentrations of ROS, which rises in LPS-activated macrophages and eventually triggers apoptosis ${ }^{45}$.

We see that during the confocal microscopy study $\mathrm{OMe}$ functionalized P1 particles shrink and the fluorescence signal disappears after $\sim 11.5$ h (see Fig. 4a). Similarly, the P3 particles are degraded; however, here some of the particles disintegrate even at earlier points in time (see Fig. 4c). We account this faster degradation to the improved solubility of $\mathbf{P 3}$-fragments in aqueous media mediated through the OEG functionalization. By contrast, the non-degradable particles do not disintegrate and no reduction in size is observable (see Fig. $4 \mathrm{~b}, \mathrm{c}$ and Supplementary Fig. 10). This proves that our imidazole functionalized CPNs are degradable in macrophages at biologically relevant levels of ROS.

Such particles could now in principle be used for imaging of malignant tumors, which are rich in tumor-associated macrophages (TAMs) ${ }^{46}$. The particles can accumulate in the tumor tissue through the enhanced permeability and retention effect. Here, they could then be taken up and degraded by the TAMs. For specific targeting of TAMs by the particles, the folic acid motif could be used to target the folate receptor $\beta$, which is expressed on TAMs ${ }^{47}$. Here, we show that we can functionalize the surface of our degradable CPNs with cysteine-terminated folic acid recognition motifs using thiol-yne click-chemistry (see Fig. 5 b) ${ }^{12}$. We continue with the OEG functionalized P3 particles and perform the attachment of cysteine functionalized folic acid to the surface of the particles in a mixture of degassed dimethyl sulfoxide and water. The folic acid compound is added 
a

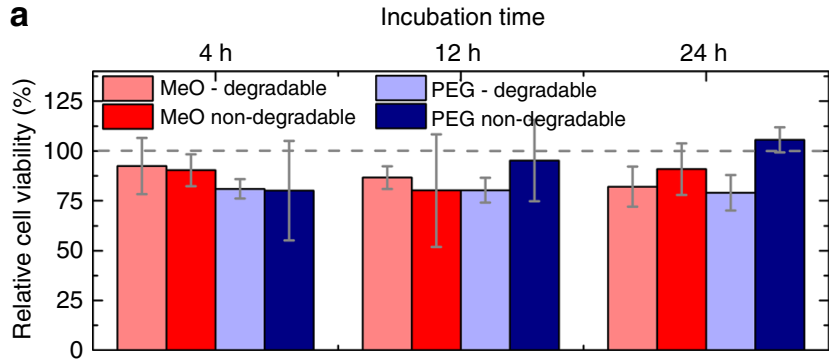

b

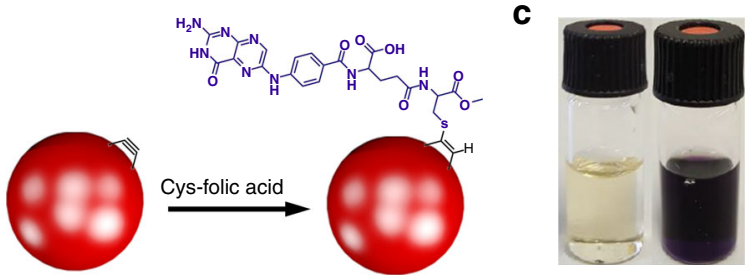

d

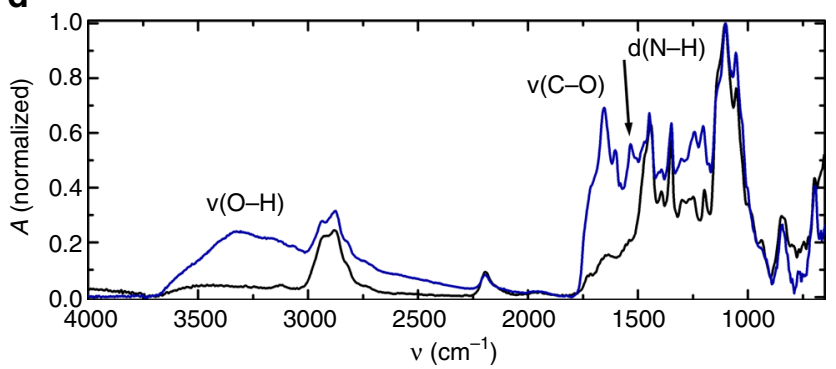

Fig. 5 Biocompatibility of the conjugated polymer nanoparticles. a Cytotoxicity test: J774A.1 cells are incubated for 4, 12, and $24 \mathrm{~h}$ with LPS (particle concentration $5 \mu \mathrm{g} / \mathrm{ml}$ ). b Acetylene units on the particles are functionalized with cysteine folate using thiol-yne click chemistry. c Kaiser test before (colorless) and after (dark-purple) surface functionalization. The dark-purple color illustrates the presence of primary amines. $\mathbf{d}$ IR spectra before (black) and after (blue) functionalization. The characteristic band of the amide oscillation is highlighted by an arrow

in large molar excess and the mixture is stirred while irradiating with $365 \mathrm{~nm}$ ultraviolet (UV) light from an $8 \mathrm{~W}$ hand lamp to allow thiol-yne click coupling. The particles are then purified by centrifugation, exchange of the supernatant, and redispersion. To test the successful attachment of the folate motif to the surface of the particles, we perform the Kaiser-test to probe for primary amines, which are part of the folic acid compound. We purify the particles until the Kaiser-test is negative for the supernatant. Then we add the Kaiser-reagent to the aqueous dispersions of our P3 particles, which turns dark purple, proving the existence of free amines on the particle surface (see inset Fig. 5b). To confirm our assumption, that this observation is due to the surface-attached folic acid, we perform IR spectroscopy on our particles before and after folic acid functionalization. We find characteristic vibrational bands for hydroxyl $\left(3324 \mathrm{~cm}^{-1}\right)$, amide carbonyl $\left(1654 \mathrm{~cm}^{-1}\right)$, and amide N-H $\left(1535 \mathrm{~cm}^{-1}\right)$ groups, which are absent in the nonfunctionalized particles (see Fig. 5b). Furthermore, we determine the $\zeta$-potential of the particles before $(-3.84 \mathrm{mV})$ and after functionalization with the folic acid moiety $(-8.71 \mathrm{mV})$. We perform the measurements at high $\mathrm{pH}$ to deprotonate the carboxylic acid moiety. Using the formula derived by Oshima et al. ${ }^{48}$ for the surface charge density of sub-micron polymer particles and assuming that each folic acid molecule contributes one negative charge, we obtain a particle coverage of $\sim 1000$ folic acid molecules per particle ( 1 molecule per $\left.250 \mathrm{~nm}^{2}\right)$. These results substantiate our claim for surface functionalization.

\section{Discussion}

In conclusion, we have developed a concept to provide fully conjugated polymer particles with bio-degradability, through integration of imidazole units. The ROS concentration in stimulated macrophages is sufficient to degrade the particles. The particles can be surface functionalized for labeling of specific biological recognition motifs for targeted imaging. In the future, it needs to be confirmed that the ROS concentration for degradation of the particles is sufficient in vivo. The concept of oxidative degradation could be extended by loading the hydrophobic conjugated polymer particles with drugs, which can be released upon degradation of the particles by macrophages at a specific site. In contrast to fluorescent degradable inorganic materials, the conjugated polymer particles provide more optical gain because of the dense luminophore packing and, therefore, have improved signal-to-noise ratios 49,50 . The approach opens a wide variety of applications for conjugated polymer particles in the biological and medical fields such as imaging, drug-delivery, and theranostics.

\section{Methods}

Dynamic light scattering. The DLS measurements are performed at $25^{\circ} \mathrm{C}$ on a Zetasizer Nano ZS from Malvern Instruments. The samples are prepared by diluting $100 \mu \mathrm{l}$ of particle dispersions with $5 \mathrm{ml}$ of deionized water. The cumulants analysis of the autocorrelation function obtained by the DLS measurements is defined by the International Standard on Dynamic Light Scattering ISO13321 and ISO22412. This analysis provides a mean value for the size ( $\mathrm{z}$-average) and a width parameter for the polydispersity index. Every determined diameter and dispersity value has been averaged over three measurements.

Gel permeation-size exclusion chromatography (SEC). Molecular weights and molecular weight distributions were determined by SEC. SEC analyses were carried out with tetrahydrofuran as eluent using a HPLC pump (PU-2080plus, Jasco) equipped with a refractive index detector (RI-2031plus, Jasco) and an evaporative light scattering detector (PL-ELS-1000, Polymer Laboratories). The sample solvent contained $250 \mathrm{mg} / \mathrm{ml} \mathrm{3,5-di-tert-4-butylhydroxytoluene} \mathrm{(} \geq 99 \%$, Fluka) as a stabilizer for THF and as an internal standard. One pre-column $(8 \times 50 \mathrm{~mm})$ and four SDplus gel columns $(8 \times 300 \mathrm{~mm}, \mathrm{MZ}$ Analysentechnik) were applied at a flow rate of $1.0 \mathrm{ml} / \mathrm{min}$ at $20^{\circ} \mathrm{C}$. The diameter of the gel particles measured $5 \mu \mathrm{m}$, the nominal pore widths were $50,10^{2}, 10^{3}$, and $10^{4} \AA$. Calibration was achieved using polystyrene standards (Polymer Standards Service).

Mass spectrometry. Mass spectra are acquired on a Finnigan SSQ7000 (EI, $70 \mathrm{eV}$ ) spectrometer and on a ThermoFinnigan LCQ Deca XP plus (ESI) spectrometer.

Kaiser test. The Kaiser test is performed by preparing the following three agents: (a) aqueous KCN solution $(1.0 \mathrm{ml}, \beta=0.66 \mathrm{mg} / \mathrm{ml})$ in pyridine $(49.0 \mathrm{ml})(\mathrm{b})$ ninhydrin $(1.0 \mathrm{mg}, 5.61 \mathrm{mmol})$ in $n$-butanol $(20.0 \mathrm{ml})(\mathrm{c})$ phenol $(20.0 \mathrm{~g}, 0.213$ $\mathrm{mol})$ in $n$-butanol $(10.0 \mathrm{ml}) \mathrm{A}$ few droplets of each agent are added to an alkaline sample and the mixture is heated to $80^{\circ} \mathrm{C}$ for a few minutes. The presence of primary amino groups is indicated by a dark blue to purple color. (In case of a negative test, the mixture remains yellow).

Confocal microscopy. Confocal laser scanning microscopy is performed on a Leica TCS SP8 to visualize the time-resolved degradation of the conjugated particles as well as the in-vitro cell experiments using prepared macrophages. The conjugated particles are excited with the wavelength of $\lambda=561 \mathrm{~nm}$. The sample chamber is the well of a 96-well microplate for the time-resolved degradation with $\mathrm{H}_{2} \mathrm{O}_{2}(3 \mathrm{M})$. For the time-resolved degradation by macrophages, the sample chamber is the well of a Cellstar cell culture flask (Greiner), which is then placed in a TOKAI HIT stage top incubator (INUBG2E-GSI) so that a stable $5 \% \mathrm{CO}_{2}+95 \%$ air mixed gas supply as well as a temperature of $37.5^{\circ} \mathrm{C}$ can be adjusted. For long-term experiments the well is temporary sealed with parafilm.

Microscopic macrophage degradation study. J774A.1 (ATCC) cells are seeded in Cellstar cell culture flasks (Greiner) and cultured in FluoroBrite (Biochrom) $+10 \%$ FBS (Invitrogen) $+1 \%$ penicillin/streptomycin (Invitrogen). Stimulation of cells is achieved by adding LPS (Sigma Aldrich) $1 \mu \mathrm{g} / \mathrm{ml}$. The cells are incubated with the degradable and non-degradable particle dispersions $(4 \mu \mathrm{g} / \mathrm{ml})$ for two hours and subsequently the dispersions are exchanged with cell culture medium. The cells are stained with CellTracker ${ }^{\mathrm{TM}}$ Blue CHMC Dye (Thermo Fisher). 
Cytotoxicity. The toxicity of the particles and their degradation products on J774A.1 is investigated by trypan blue staining. In all, $3 \times 10^{4}$ cells are seeded in 24-well plates (Corning) and cultured in Fluorobrite (Biochrom) $+10 \%$ FBS (Invitrogen) $+1 \%$ penicillin/streptomycin (Invitrogen) and stimulated by adding 1 $\mu \mathrm{g} / \mathrm{ml}$ LPS (Sigma Aldrich) for $1 \mathrm{~h}$. Cells incubated with cell culture medium without LPS served as negative controls. Degradable and non-degradable particles are diluted in cell culture medium and added to the cells with a concentration of either 5 or $100 \mu \mathrm{g} / \mathrm{ml}$ and the cells are incubated for $4,12,24$, and $48 \mathrm{~h}$. Three samples per condition are analyzed. After incubation, the cells are removed with a cell scraper (Falcon) and transferred to eppendorf tubes. The cell suspension is centrifuged and the supernatant is removed. The cell pellet was dissolved in $50 \mu \mathrm{l}$ cell culture medium. Twenty-five microliters of the cell suspension is mixed with $25 \mu \mathrm{l}$ Trypan blue staining solution (Gibco). Trypan blue positive cells (blue cells) were counted using Cedex XS cell counter (Innovates AG). The percentage of positive cells as a function of the total cell number was calculated.

Photoluminescence spectroscopy and quantum yield $(\Phi)$. Photoluminescence spectroscopy measurements are conducted on a Horiba Jobin-Yvon FluoroMax4 spectrofluorometer with a $150 \mathrm{~W}$ xenon lamp, ozone free. The software has been FluorEssenceTM version 3.8.2.2, Origin version 8.6001.The conjugated particles are dispersed in water and in case of the degradation studies $\mathrm{H}_{2} \mathrm{O}_{2}$ is added, leading to a $\mathrm{H}_{2} \mathrm{O}_{2}$-concentration of $1.1 \mathrm{wt} \%$.

UV-Visible spectroscopy. Extinctions are measured with a Varian Cary 50 Bio UV-Visible spectrophotometer. The software is Varian UV Cary Scan 3.00(303).

Scanning electron microscopy. Field emission scanning electron microscopy is performed on a S- 4800 microscope (Hitachi) to determine the size and shape of the synthesized conjugated particles. Before imaging, particle dispersions are deposited and dried on silicon wafer (CrysTec $\mathrm{GmbH}$ ). All samples are sputtered with a thin layer of $\mathrm{Au} / \mathrm{Pd}$.

Data availability. All data are available from the corresponding authors upon reasonable request.

Received: 6 February 2017 Accepted: 5 July 2017

Published online: 07 September 2017

\section{References}

1. Wu, C. \& Chiu, D. T. Highly fluorescent semiconducting polymer dots for biology and medicine. Angew. Chemie Int. Ed. 52, 2-26 (2013).

2. $\mathrm{Pu}, \mathrm{K}$., Shuhendler, A. J. \& Rao, J. Semiconducting polymer nanoprobe for in vivo imaging of reactive oxygen and nitrogen species. Angew. Chemie Int. Ed. 52, 10325-10329 (2013).

3. Chan, Y.-H. \& Wu, P.-J. Semiconducting polymer nanoparticles as fluorescent probes for biological imaging and sensing. Part. Part. Syst. Charact. 32, 11-28 (2015).

4. Feng, L. et al. Conjugated polymer nanoparticles: preparation, properties, functionalization and biological applications. Chem. Soc. Rev. 42, 6620-6633 (2013).

5. Wu, C., Bull, B., Szymanski, C., Christensen, K. \& McNeill, J. Multicolor conjugated polymer dots for biological fluorescence imaging. ACS Nano 2 , 2415-2423 (2008)

6. Rahim, N. A. A. et al. Conjugated polymer nanoparticles for two-photon imaging of endothelial cells in a tissue model. Adv. Mater. 21, 3492-3496 (2009).

7. $\mathrm{Pu}, \mathrm{K}$. et al. Semiconducting polymer nanoparticles as photoacoustic molecular imaging probes in living mice. Nat. Nanotechnol. 9, 233-239 (2014).

8. Fernando, L. P. et al. Mechanism of cellular uptake of highly fluorescent conjugated polymer nanoparticles. Biomacromolecules 11, 2675-2682 (2010).

9. Palner, M., Pu, K., Shao, S. \& Rao, J. Semiconducting polymer nanoparticles with persistent near-infrared luminescence for in vivo optical imaging. Angew. Chemie Int. Ed. 127, 11639-11642 (2015).

10. Li, K. \& Liu, B. Polymer-encapsulated organic nanoparticles for fluorescence and photoacoustic imaging. Chem. Soc. Rev. 43, 6570-6597 (2014).

11. Feng, G. et al. Multifunctional conjugated polymer nanoparticles for image-guided photodynamic and photothermal therapy. Small 13, 1602807 (2016).

12. Anwar, N., Rix, A., Lederle, W. \& Kuehne, A. J. C. RGD-decorated conjugated polymer particles as fluorescent biomedical probes prepared by Sonogashira dispersion polymerization. Chem. Commun. 51, 9358-9361 (2015).

13. Wu, C. et al. Ultrabright and bioorthogonal labeling of cellular targets using semiconducting polymer dots and click chemistry. Angew. Chemie Int. Ed. 49, 9436-9440 (2010)
14. Feng, L., Liu, L., Lv, F., Bazan, G. C. \& Wang, S. Preparation and biofunctionalization of multicolor conjugated polymer nanoparticles for imaging and detection of tumor cells. Adv. Mater. 26, 3926-3930 (2014).

15. Ahmed, E., Morton, S. W., Hammond, P. T. \& Swager, T. M. Fluorescent multiblock $\pi$-conjugated polymer nanoparticles for in vivo tumor targeting. Adv. Mater. 25, 4504-4510 (2013).

16. Wu, C. et al. Design of highly emissive polymer dot bioconjugates for in vivo tumor targeting. Angew. Chem. Int. Ed. Engl. 50, 3430-3434 (2011).

17. Kuehne, A. J. C., Gather, M. C. \& Sprakel, J. Monodisperse conjugated polymer particles by Suzuki-Miyaura dispersion polymerization. Nat. Commun. 3, 1088 (2012).

18. Ciftci, S. \& Kuehne, A. J. C. Monodisperse conjugated polymer particles via heck coupling - a kinetic study to unravel particle formation in step-growth dispersion polymerization. Macromolecules. 48, 8389-8393 (2015).

19. Mikosch, A., Ciftci, S. \& Kuehne, A. J. C. Colloidal crystal lasers from monodisperse conjugated polymer particles via bottom-up coassembly in a sol -gel matrix. ACS Nano 10, 10195-10201 (2016).

20. Chen, T. et al. Monodisperse AIE-active conjugated polymer nanoparticles via dispersion polymerization using geminal cross-coupling of 1,1-dibromoolefins. Small 12, 6547-6552 (2016).

21. Parrenin, L., Brochon, C., Hadziiannou, G. \& Cloutet, E. Low bandgap semiconducting copolymer nanoparticles by suzuki cross-coupling polymerization in alcoholic dispersed media. Macromol. Rapid Commun. 36, 1816-1821 (2015).

22. Landfester, K. et al. Semiconducting polymer nanospheres in aqueous dispersion prepared by a miniemulsion process. Adv. Mater. 14, 651 (2002).

23. Baier, M. C., Huber, J. \& Mecking, S. Fluorescent conjugated polymer nanoparticles by polymerization in miniemulsion. J. Am. Chem. Soc. 131, 14267-14273 (2009).

24. Herranz-Blanco, B. et al. $\mathrm{pH}$-switch nanoprecipitation of polymeric nanoparticles for multimodal cancer targeting and intracellular triggered delivery of doxorubicin. Adv. Healthc. Mater 5, 1904-1916 (2016).

25. Wu, C., Szymanski, C. \& McNeill, J. Preparation and encapsulation of highly fluorescent conjugated polymer nanoparticles. Langmuir 22, 2956-2960 (2006).

26. Longmire, M., Choyke, P. L. \& Kobayashi, H. Clearance properties of nano-sized particles and molecules as imaging agents: considerations and caveats. Nanomedicine 3, 703-717 (2008)

27. Blanco, E., Shen, H. \& Ferrari, M. Principles of nanoparticle design for overcoming biological barriers to drug delivery. Nat. Biotechnol. 33, 941-951 (2015).

28. Medina, C., Santos-Martinez, M., Radomski, A., Corrigan, O. \& Radomski, M. Nanoparticles: pharmacological and toxicological significance. Br. J. Pharmacol. 150, 552-558 (2007).

29. Lammers, T., Kiessling, F., Hennink, W. E. \& Storm, G. Nanotheranostics and image-guided drug delivery: Current concepts and future directions. Mol. Pharm. 7, 1899-1912 (2010).

30. Panyam, J. \& Labhasetwar, V. Biodegradable nanoparticles for drug and gene delivery to cells and tissue. Adv. Drug Deliv. Rev. 55, 329-347 (2003).

31. Tian, H., Tang, Z., Zhuang, X., Chen, X. \& Jing, X. Biodegradable synthetic polymers: Preparation, functionalization and biomedical application. Prog. Polym. Sci. 37, 237-280 (2012)

32. Oba, Y., Kato, S., Ojika, M. \& Inouye, S. Biosynthesis of coelenterazine in the deep-sea copepod, Metridia pacifica. Biochem. Biophys. Res. Commun. 390, 684-688 (2009).

33. Francis, W. R., Shaner, N. C., Christianson, L. M., Powers, M. L. \& Haddock, S. H. D. Occurrence of isopenicillin-N-synthase homologs in bioluminescent ctenophores and implications for coelenterazine biosynthesis. PLOS ONE 10, 1-20 (2015).

34. Nishiuchi, Y. et al. Chemical synthesis of the precursor molecule of the Aequorea green fluorescent protein, subsequent folding, and development of fluorescence. Proc. Natl Acad. Sci. 95, 13549-13554 (1998).

35. Rorijea, E., Germaa, F., Philippb, B., Schink, B. \& Beimborna, D. B. Prediction of biodegradability from structure: Imidazoles. SAR QSAR Environ. Res. 13, 199-204 (2002).

36. Klepp, J., Fallert-Müller, A., Grimm, K., Hull, W. E. \& Rétey, J. Mechanism of action of urocanase. Eur. J. Biochem. 192, 669-676 (1990).

37. Davies, M. J. Reactive species formed on proteins exposed to singlet oxygen. Photochem. Photobiol. Sci. 3, 17-25 (2004).

38. Forman, H. J. \& Torres, M. Reactive oxygen species and cell signaling: Respiratory burst in macrophage signaling. Am. J. Respir. Crit. Care Med. 166, S4-S8 (2002)

39. Carreras, M., Pargament, G., Catz, S., Poderoso, J. \& Boveris, A. Kinetics of nitric oxide and hydrogen peroxide production and formation of peroxynitrite during the respiratory burst of human neutrophils. FEBS Lett. 341, 65-68 (1994).

40. Barrett, K. E. J. Dispersion polymerisation in organic media. Br. Polym. J. 5, 259-271 (1973). 
41. Rosencrantz, R. R., Rahimi, K. \& Kuehne, A. J. C. Morphology control in poly (9,9-di-n-octyl-2,7-fluorene) spherulite particles prepared via dispersion polymerization. J. Phys. Chem. B. 118, 6324-6328 (2014).

42. Jenekhe, S. A. \& Osaheni, J. A. Excimers and exciplexes of conjugated polymers. Science (80-.) 265, 765-768 (1994).

43. Thomas, R. et al. The influence of crystal packing on the solid state fluorescence behavior of alkyloxy substituted phenyleneethynylenes. J. Mater. Chem. 19, 4401 (2009).

44. Botiz, I. et al. Enhancing the photoluminescence emission of conjugated MEH-PPV by light processing. ACS Appl. Mater. Interfaces 6, 4974-4979 (2014).

45. Matés, J. M. \& Sánchez-Jiménez, F. M. Role of reactive oxygen species in apoptosis: Implications for cancer therapy. Int. J. Biochem. Cell Biol. 32, 157-170 (2000).

46. Noy, R. \& Pollard, J. W. Tumor-associated macrophages: from mechanisms to therapy. Immunity 41, 49-61 (2014).

47. Puig-Kröger, A. et al. Folate receptor $\beta$ is expressed by tumor-associated macrophages and constitutes a marker for M2 anti-inflammatory/regulatory macrophages. Cancer Res. 69, 9395-9403 (2009).

48. Ohsawa, K., Murata, M. \& Ohshima, H. Zeta potential and surface charge density of polystyrene-latex; comparison with synaptic vesicle and brush border membrane vesicle. Colloid Polym. Sci. 264, 1005-1009 (2005).

49. Chen, F. et al. In vivo tumor targeting and image-guided drug delivery with antibody-conjugated, radiolabeled mesoporous silica nanoparticles. ACS Nano 7, 9027-9039 (2013).

50. Park, J.-H. et al. Biodegradable luminescent porous silicon nanoparticles for in vivo applications. Nat. Mater. 8, 331-336 (2009).

\section{Acknowledgements}

We thankfully acknowledge funding from the BMBF in form of the AktiPhotoPol research group (Grant No. 13N13522) and of LiSyM (Grant No. 031L0041). This work was performed in part at the Center for Chemical Polymer Technology CPT, which was supported by the EU and the federal state of North Rhine-Westphalia (Grant EFRE 3000883 02).

\section{Author contributions}

A.J.C.K. conceived the idea of the project, A.J.C.K. and W.L. planned the experiments, T.R. and S.L. performed particle synthesis and characterization, D.G. and T.R. performed confocal microscopy experiments, A.R. performed cell culture experiments. T.R., A.R., W.L., and A.J.C.K. wrote the initial manuscript, which all authors (T.R., A.R., S.L., D.G., F.K., W.L., and A.J.C.K.) reviewed and edited.

\section{Additional information}

Supplementary Information accompanies this paper at doi:10.1038/s41467-017-00545-0.

Competing interests: The authors declare no competing financial interests.

Reprints and permission information is available online at http://npg.nature.com/ reprintsandpermissions/

Publisher's note: Springer Nature remains neutral with regard to jurisdictional claims in published maps and institutional affiliations.

\section{(c) (i)}

Open Access This article is licensed under a Creative Commons Attribution 4.0 International License, which permits use, sharing, adaptation, distribution and reproduction in any medium or format, as long as you give appropriate credit to the original author(s) and the source, provide a link to the Creative Commons license, and indicate if changes were made. The images or other third party material in this article are included in the article's Creative Commons license, unless indicated otherwise in a credit line to the material. If material is not included in the article's Creative Commons license and your intended use is not permitted by statutory regulation or exceeds the permitted use, you will need to obtain permission directly from the copyright holder. To view a copy of this license, visit http://creativecommons.org/ licenses/by/4.0/.

(c) The Author(s) 2017 